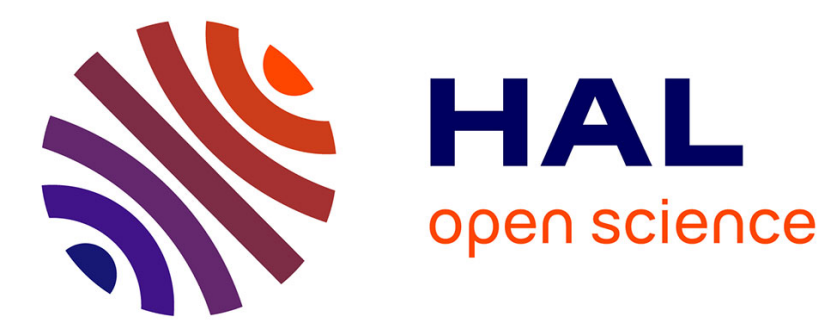

\title{
DÉFAUTS COLLOÏDAUX CARACTÉRISANT LES PARTICULES IMPLANTÉES DANS LES HALOGÉNURES ALCALINS
}

\author{
P. Thévenard, J. Davenas, A. Perez, C. Dupuy
}

\section{- To cite this version:}

P. Thévenard, J. Davenas, A. Perez, C. Dupuy. DÉFAUTS COLLOÏDAUX CARACTÉRISANT LES PARTICULES IMPLANTÉES DANS LES HALOGÉNURES ALCALINS. Journal de Physique Colloques, 1973, 34 (C5), pp.C5-79-C5-82. 10.1051/jphyscol:1973515 . jpa-00215302

HAL Id: jpa-00215302 https://hal.science/jpa-00215302

Submitted on 1 Jan 1973

HAL is a multi-disciplinary open access archive for the deposit and dissemination of scientific research documents, whether they are published or not. The documents may come from teaching and research institutions in France or abroad, or from public or private research centers.
L'archive ouverte pluridisciplinaire HAL, est destinée au dépôt et à la diffusion de documents scientifiques de niveau recherche, publiés ou non, émanant des établissements d'enseignement et de recherche français ou étrangers, des laboratoires publics ou privés. 


\title{
DÉFAUTS COLLOÏDAUX CARACTÉRISANT LES PARTICULES IMPLANTÉES DANS LES HALOGÉNURES ALCALINS
}

\author{
P. THÉVENARD, J. DAVENAS, A. PEREZ et C. H. S. DUPUY \\ Département de Physique des Matériaux, Associé au CNRS \\ Université Claude-Bernard, Lyon I, 69621 Villeurbanne, France
}

\begin{abstract}
Résumé. - Les défauts créés dans LiF par bombardement avec des ions alcalins et halogènes ont été étudiés par des mesures d'absorption optique. Des traitements thermiques révèlent de nouvelles bandes d'absorption dont les positions dépendent de la nature de l'ion implanté. Dans le cas d'implantation d'ions alcalins, les bandes d'absorption obtenues obéissent à la théorie de Doyle qui montre qu'elles résultent de la résonance de plasma de particules métalliques formées par la coagulation des ions implantés. Dans le cas d'implantation d'ions halogènes, les bandes d'absorption ont une position qui coïncide avec les bandes d'absorption de molécules d'halogènes, alors que dans le cas de protons ou de deutérium, les bandes obtenues ne peuvent pas s'expliquer par un caractère moléculaire.

Abstract. - The defects created in $\mathrm{LiF}$ by bombardment with alkali and halogen ions have been studied by optical absorption measurements. New absorption bands appear after thermal treatments, their wavelength positions depend on the nature of the implanted ions. In the case of alkali ion implantation, the absorption band positions, following Doyle's theory, are related to the plasma resonance of metallic particles which proceed from the agglomeration of the implanted ions. In the case of halogen ion implantation, the absorption band positions are in good agreement with the absorption bands of halogen molecules, but with proton or deuteron implantation, the absorption band positions cannot be explained by a molecular character.
\end{abstract}

1. Introduction. - Les ions ayant une énergie de l'ordre du $\mathrm{MeV}$ créent dans les halogénures alcalins divers types de défauts qui colorent ces cristaux. Ils peuvent être classés d'après les processus en : défauts d'ionisation, défauts de chocs et défauts caractéristiques de la particule implantée. La faible pénétration de ces ions $(<$ à $10 \mu)$ permet de mesurer optiquement de grandes concentrations de défauts $\left(\sim 10^{20}\right.$ défauts par $\left.\mathrm{cm}^{3}\right)$.

Les principaux défauts connus sont essentiellement créés par des mécanismes d'ionisation alors que les défauts résultant des chocs de la particule incidente avec les ions du réseau ont été très peu étudiés. De plus, pour des doses d'irradiation suffisantes, nous pouvons espérer mettre en évidence des bandes d'absorption caractéristiques de défauts liés aux particules implantées. En conséquence, nous avons effectué deux types d'irradiation, d'une part, différents halogénures alcalins $(\mathrm{LiF}, \mathrm{NaCl}, \mathrm{KCl}, \mathrm{KBr})$ ont été bombardés avec une même particule et d'autre part, un même halogénure alcalin $(\mathrm{LiF})$ a été irradié avec des ions différents : proton, alcalins $\left(\mathrm{Li}^{+}\right.$, $\left.\mathrm{Na}^{+}, \mathrm{K}^{+}\right)$, oxygène et halogènes $\left(\mathrm{F}^{6+}\right.$ et $\left.\mathrm{Cl}^{8+}\right)$. Immédiatement après l'irradiation, aucune bande caractéristique des ions implantés n'apparaît dans les spectres d'absorption optique. Par contre, des traitements thermiques favorisant l'agglomération de ces ions font apparaittre ces bandes, alors que les centres du type $\mathrm{F}$ et agglomérats de $\mathrm{F}$ déjà présents disparaissent.

2. Techniques expérimentales. - Les cibles utilisées sont des lamelles monocristallines de $\mathrm{LiF}$, $\mathrm{NaCl}, \mathrm{KCl}$ et $\mathrm{KBr}$ clivées à partir de blocs provenant de " quartz et silice ». Les irradiations avec des ions $\mathrm{Li}^{+}$de $2 \mathrm{MeV}, \mathrm{Na}^{+}$de $0,8 \mathrm{MeV}, \mathrm{K}^{+}$de $0,5 \mathrm{MeV}$, $\mathrm{H}^{+}$de $2 \mathrm{MeV}$ et $\mathrm{D}^{+}$de $2 \mathrm{MeV}$ ont été effectuées à l'aide des accélérateurs Van de Graaf de l'Institut de Physique Nucléaire et du Laboratoire de Physique de l'Atmosphère de Lyon. Les ions $\mathrm{Cl}^{8+}$ de $67 \mathrm{MeV}$, $\mathrm{O}^{7+}$ de $60 \mathrm{MeV}$ et $\mathrm{F}^{6+}$ de $51 \mathrm{MeV}$ ont été obtenus avec l'accélérateur tandem du Département de Physique des Basses Energies du CEN de Saclay. Toutes les irradiations ont été faites à la température ambiante. Les flux de particules utilisées étaient suffisamment faibles $\left(\sim 10^{10}\right.$ ions. $\left.\mathrm{cm}^{-2} \cdot \mathrm{s}^{-1}\right)$ pour éviter un échauffement trop important et une détérioration des cibles pendant le bombardement. Les doses totales d'ions implantés étaient comprises entre $10^{10}$ et $10^{16}$ ions. $\mathrm{cm}^{-2}$. Les traitements thermiques nécessaires à l'observation des défauts liés aux particules implantées ont été réalisés à la pression normale pour des températures comprises entre $100{ }^{\circ} \mathrm{C}$ et $400{ }^{\circ} \mathrm{C}$ suivant les halogénures alcalins. 
L'absorption optique des échantillons irradiés a été mesurée avec un spectrophotomètre à double faisceau « Beckman DK 2a ».

3. Résultats. - 3.1 Cibles DIFFÉRENTES IRRADIÉES AVEC UNE MÊME PARTICULE. - Immédiatement après irradiation, les spectres d'absorption optique des cristaux de $\mathrm{LiF}, \mathrm{NaCl}, \mathrm{KCl}$, et $\mathrm{KBr}$, bombardés avec environ $10^{14}$ ions $\mathrm{O}^{7+}$ par $\mathrm{cm}^{2}$, sont composés des bandes caractéristiques des centres $\mathrm{F}$, agglomérats de $F$ et $V_{n}$.

Des traitements thermiques de une heure effectués à des températures de $300^{\circ} \mathrm{C}$ pour $\mathrm{LiF}, 220^{\circ} \mathrm{C}$ pour $\mathrm{NaCl}, 200{ }^{\circ} \mathrm{C}$ pour $\mathrm{KCl}$ et $170{ }^{\circ} \mathrm{C}$ pour $\mathrm{KBr}$, font apparaître les bandes d'absorption des colloïdes du métal alcalin de la matrice ; les positions en longueur d'onde de ces bandes sont données dans le tableau I.

\section{TABLEAU I}

\begin{tabular}{|c|c|c|c|c|}
\hline & $C_{L i}^{L_{i} i F}$ & $\mathrm{C}_{\mathrm{Na}}^{\mathrm{NaCl}}$ & $\mathrm{C}_{\mathrm{K}}^{\mathrm{KCl}}$ & $\mathrm{C}_{\mathrm{K}}^{\mathrm{KBr}}$ \\
\hline$\lambda \AA$ & 4450 & 5700 & 7700 & 8250 \\
\hline
\end{tabular}

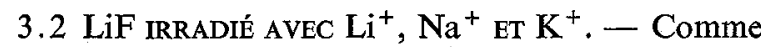
dans le cas précédent, immédiatement après irradiation nous n'observons que les centres $F$ et agglomérats de $F$. Sur les figures 1 et 2 nous voyons l'évolution

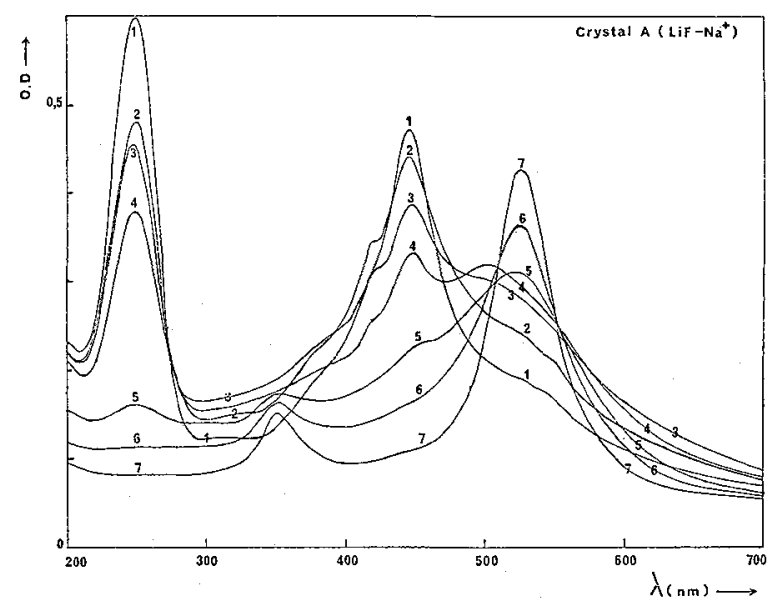

Fig. 1. - Evolution du spectre d'absorption optique d'un échantillon de LiF irradié à la température ambiante avec 1016 ions $\mathrm{Na}^{+} / \mathrm{cm}^{2}$ (1) puis successivement recuit à $140{ }^{\circ} \mathrm{C}$ pendant $75 \mathrm{~min}, 160^{\circ} \mathrm{C}, 105 \min (2), 180^{\circ} \mathrm{C}, 105 \mathrm{~min}, 200^{\circ} \mathrm{C}$, $105 \mathrm{~min}(3), 220^{\circ} \mathrm{C}, 105 \mathrm{~min}, 240^{\circ} \mathrm{C}, 105 \mathrm{~min}(4), 260^{\circ} \mathrm{C}, 105 \mathrm{~min}$, $300{ }^{\circ} \mathrm{C}, 105 \mathrm{~min}(5), 340{ }^{\circ} \mathrm{C}, 105 \mathrm{~min}(6), 380{ }^{\circ} \mathrm{C}, 105 \mathrm{~min}$ et $420^{\circ} \mathrm{C}, 105 \min (7)$.

des spectres d'absorption optique en fonction des traitements thermiques. Dans un premier stade, pour des températures de recuits inférieures à $200^{\circ} \mathrm{C}$ nous voyons disparaître les centres $F$ et $F_{n}$ alors que deux bandes situées à $4180 \AA \AA$ et $5000 \AA$ associées à des pré-précipités de lithium [1] augmentent en amplitude. Dans un deuxième stade, pour des tem-

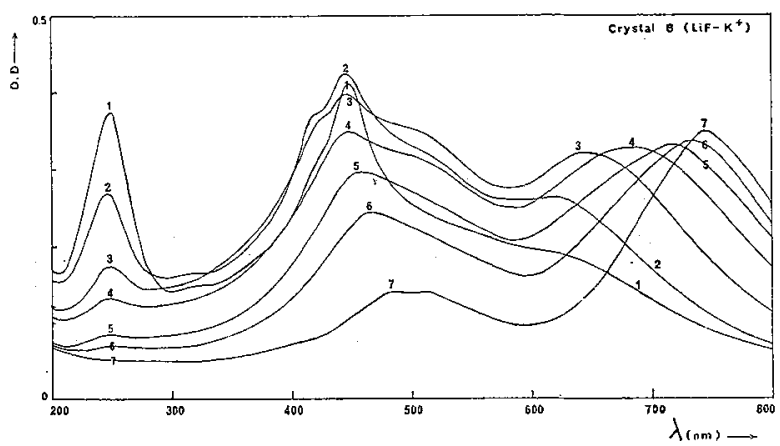

Fig. 2. - Evolution du spectre d'absorption d'un échantillon de LiF irradié à la température ambiante avec $7 \times 10^{15}$ ions $\mathrm{K}^{+} / \mathrm{cm}^{2}$ puis ensuite recuit comme cela est indiqué figure 1 .

pératures de recuits supérieures à $200{ }^{\circ} \mathrm{C}$, la bande colloïdale du lithium $\left(\mathrm{C}_{\mathrm{Li}}^{\mathrm{LiF}}: 4450 \AA\right)$ déjà signalée dans le paragraphe précédent, est présente. Elle possède une amplitude plus importante dans le cas de l'irradiation avec des ions $\mathrm{Li}^{+}$. Cette bande a également été observée dans LiF irradié avec d'autres particules [2], [3], [4], [5]. Pour les cristaux bombardés avec $\mathrm{Na}^{+}$ et $\mathrm{K}^{+}$, on voit apparaître et augmenter en amplitude une bande d'absorption dont la position en longueur d'onde dépend de l'ion implanté (Tableau II).

\section{TABLEAU II}

$$
\overline{\lambda \AA} \quad \frac{\mathrm{C}_{\mathrm{Li}}^{\mathrm{LiF}}}{4 \overline{450}} \quad \frac{\mathrm{C}_{\mathrm{Na}}^{\mathrm{LiF}}}{5 \overline{200}} \quad \frac{6 \overline{\mathrm{C}}}{\mathrm{C}_{\mathrm{K}}^{\mathrm{LiF}}}
$$

L'apparition de ces bandes dépend fortement de la technique de recuit. Dans certains cas nous avons obtenu des bandes qui croissaient à longueur d'onde fixe alors que dans d'autres cas, ces bandes se déplaçaient vers les grandes longueurs d'onde avant d'atteindre leur amplitude maximum.

3.3 LiF IRRADIÉ AVEC $\mathrm{F}^{6+}, \mathrm{Cl}^{8+}, \mathrm{H}^{+}$eT $\mathrm{D}^{+} .-$ Les traitements thermiques effectués à $200^{\circ} \mathrm{C}$ pendant 15 min permettent d'obtenir les bandes d'absorption associées à ces particules implantées, leurs positions sont données dans le tableau III.

\section{TABLeau III}

$$
\overline{\lambda \AA} \quad 2 \frac{C_{F}^{L i F}}{2 \overline{900}} \quad \frac{C_{\mathrm{Cl}}^{\mathrm{LiF}}}{3 \overline{300}} \quad \frac{\mathrm{C}_{\mathrm{H}}^{\mathrm{LiF}}}{3 \overline{400}} \quad \frac{\mathrm{C}_{\mathrm{D}}^{\mathrm{LiF}}}{3 \overline{500}}
$$

La bande située à $2900 \AA$ peut être attribuée au fluor, elle est observable quel que soit l'ion incident. Toutefois nous devons signaler que toutes les bandes d'absorption citées dans ce paragraphe sont assez difficiles à révéler. Leurs amplitudes sont bien plus faibles que celles obtenues avec des ions alcalins pour 
des doses d'irradiation du même ordre $\left(\sim 10^{15}\right.$ ions. $\mathrm{cm}^{-2}$ ). Les largeurs à mi-hauteur de ces bandes sont importantes $(\sim 1 \mathrm{eV})$ ce qui rend leurs résolutions encore plus difficiles. Dans certains cas $\left(\mathrm{C}_{\mathrm{Cl}}^{\mathrm{LiF}}\right.$ et $\mathrm{C}_{\mathrm{D}}^{\mathrm{LiF}}$ ) nous avons eu recours à des spectres différentiels.

4. Discussion. - Les irradiations de $\mathrm{LiF}, \mathrm{NaCl}$, $\mathrm{KCl}, \mathrm{KBr}$ font apparaître après traitements thermiques les bandes colloïdales caractéristiques du métal de la matrice. Le rendement dans la formation de ces colloïdes métalliques liés aux chocs des particules incidentes sur les ions du réseau dépend de la nature de cette particule et de la cible. Nous pouvons comparer (Tableau IV) les positions des bandes colloïdales obtenues par irradiation avec celles obtenues par coloration additive de ces cristaux dans la vapeur du métal [6].

\section{TABLEAU IV}

$\begin{array}{lccc} & \underline{\mathrm{C}_{\mathrm{Na}}^{\mathrm{NaCl}}} & \underline{\mathrm{C}_{\mathrm{K}}^{\mathrm{KCl}}} & \underline{\mathrm{C}_{\mathrm{K}}^{\mathrm{KBr}}} \\ \begin{array}{l}\lambda \AA \\ \begin{array}{c}\text { irradiation } \\ \lambda \AA\end{array}\end{array} & 5700 & 7700 & 8250 \\ \begin{array}{c}\text { Coloration } \\ \text { additive }\end{array} & 5650 & 7750 & 8500 \\ & & & \end{array}$

Nous constatons qu'il existe un assez bon accord entre ces différentes valeurs.

Doyle [7], [8] a pu interpréter les positions et les largeurs à mi-hauteur de ces bandes, dans le cas de petits colloïdes sphériques et non diffusants. Les positions sont données par la formule suivante :

$$
\lambda=\left(1+2 n^{2}\right)^{1 / 2} \lambda_{\mathrm{C}}
$$

dans laquelle $n$ est l'indice de réfraction du milieu et $\lambda_{\mathrm{C}}$ la longueur d'onde de coupure du métal alcalin formant le colloïde.

Connaissant les positions des bandes d'absorption des colloïdes de sodium dans $\mathrm{NaCl}$ et de potassium dans $\mathrm{KCl}$ nous pouvons traiter le cas des colloïdes d'ions implantés en appliquant la formule (1) et prévoir les positions de ces bandes dans $\mathrm{LiF}$.

Dans le tableau V, nous comparons ces positions calculées à celles obtenues expérimentalement dans $\mathrm{LiF}$ irradié avec $\mathrm{Na}^{+}$et $\mathrm{K}^{+}$.

\begin{tabular}{|c|c|c|}
\hline & $\mathrm{C}_{\mathrm{Na}}^{\mathrm{LiF}}$ & $\mathrm{C}_{\mathrm{K}}^{\mathrm{LiF}}$ \\
\hline$\lambda \AA$ & $5 \overline{350}$ & $7 \overline{350}$ \\
\hline $\begin{array}{l}\text { Eve par (1) } \\
\lambda \AA\end{array}$ & 5200 & 6800 \\
\hline
\end{tabular}

Les écarts constatés entre les valeurs prévues et les valeurs expérimentales peuvent être expliqués soit par des formes de colloïdes non sphériques et également par des variations de compacité des colloïdes qui interviennent dans le facteur $\lambda_{\mathrm{C}}$ par l'intermédiaire de la densité d'électrons libres. Il semble que ce dernier facteur soit le plus important. Dans la formule (1) nous pouvons expliciter $\lambda_{\mathrm{C}}$ en fonction de $N / a^{3}$. $N$ étant le nombre d'électrons libres par maille et $a$ le paramètre cristallin du colloïde :

$$
\lambda=\left(1+2 n^{2}\right)^{1 / 2} \cdot 2 \pi C\left(\frac{m^{*}}{4 \pi \mathrm{Ne}^{2}}\right)^{1 / 2} \cdot a^{3 / 2}
$$

$m^{*}$ est la masse effective de l'électron. Cette loi se vérifie mal lorsqu'on utilise les paramètres (cubique centré) de ces métaux, mais ces métaux ne sont peutêtre pas dans la phase normale. En effet, des mesures aux rayons $\mathrm{X}$ sur $\mathrm{LiF}$ irradié avec de fortes doses de neutrons [3] et sur LiF irradié aux rayons $\gamma$ [9] ont montré que les colloïdes de lithium existaient sous une phase cubique à faces centrées qui peut évoluer par recuit jusqu'à la phase cubique centrée. Ceci peut s'expliquer par des contraintes importantes exercées par la matrice sur le colloïde. Dans le cas des colloïdes de sodium et de potassium dans LiF les systèmes cristallins et les paramètres ne sont pas connus et nous sommes dans l'impossibilité de vérifier la formule (2).

Les bandes d'absorption associées au fluor et au chlore que nous observons expérimentalement sont en accord avec celles des molécules $\mathrm{F}_{2}$ et $\mathrm{Cl}_{2}$ [10]. De plus, l'absorption de ces molécules est peu dépendante du milieu dans lequel elles se trouvent. Il semblerait donc que les ions fluor et chlore passent sous forme moléculaire à la suite des traitements thermiques. Par contre, les bandes d'absorption associées à l'hydrogène et au deutérium ne peuvent être expliquées par un caractère moléculaire. Toutefois on pourrait envisager, compte tenu des propriétés particulières de l'hydrogène, qu'il puisse se mettre sous une forme à « caractère métallique ». Dans ce cas, des expériences de RPE et de RMN peuvent être envisagées pour vérifier cette hypothèse.

5. Conclusion. - A notre connaissance, le fluorure de lithium ne se colore pas additivement dans la vapeur du métal alcalin comme les autres halogénures alcalins. Les expériences d'implantation sont donc les seules qui permettent d'étudier les colloïdes métalliques dans ce cas. D'autre part, les expériences de colorations additives dans la vapeur de l'halogène sont très difficiles car elles nécessitent des pressions très importantes et ont été très peu étudiées. L'implantation est donc encore un bon moyen pour étudier ces phénomènes. De plus, les valeurs des densités optiques obtenues pour les bandes colloïdales font que l'implantation constitue une méthode intéressante pour étudier les petits amas métalliques. Enfin il serait nécessaire de faire appel à d'autres techniques telles que : $\mathrm{R}$ X et microscopic électronique pour étudier les systèmes cristallins des colloïdes métalliques. 
Remerciements. - Nous remercions M. le Professeur A. Sarazin et M. le Professeur M. Dufay pour leur assistance avec les accélérateurs Van de
Graaff. Nous remercions également M. Delaunay pour toute l'aide qu'il nous a apportée lors des irradiations au CEN de Saclay.

\section{Bibliographie}

[1] FARge, Y., Thèse, Paris (1967).

[2] Perez, A., Thevenard, P. et Dupuy, C. H. S., $C . R$. Hebd. Séan. Acad. Sci. 271B (1970) 519.

[3] Lambert, M., Thèse, Paris (1958).

[4] Radchenko, I. S., Sov. Phys. Solid State 11 (1970) 1476.

[5] Politov, N. G., Vorozheikina, F., Sov. Phys. Solid State 12 (1970) 277.
[6] Scotr, A. B., Smith, W. A., Thompson, M. A., J. Phys. Chem. 57 (1953) 757.

[7] Doyle, W. T., Phys. Rev. 111 (1958) 1067.

[8] Doyle, W. T., Proc, Phys. Soc. 75 (1960) 649.

[9] Douls, J., Conf. Cristaux Ioniques, Marseille (1972).

[10] Herzberg, G., Spectra of diatomic molecules (Ed. D. Van Nostrand Compagny, Princeton). 